\title{
Constraining planet structure and composition from stellar chemistry: trends in different stellar populations
}

\author{
N. C. Santos ${ }^{1,2}$, V. Adibekyan ${ }^{1}$, C. Dorn ${ }^{3}$, C. Mordasini ${ }^{4}$, L. Noack ${ }^{5,6}$, S. C. C. Barros ${ }^{1}$, E. Delgado-Mena ${ }^{1}$, \\ O. Demangeon ${ }^{1}$, J. P. Faria ${ }^{1,2}$, G. Israelian ${ }^{7,8}$, and S. G. Sousa ${ }^{1}$ \\ ${ }^{1}$ Instituto de Astrofísica e Ciências do Espaço, Universidade do Porto, CAUP, Rua das Estrelas, 4150-762 Porto, Portugal \\ e-mail: nuno@astro.up.pt \\ 2 Departamento de Física e Astronomia, Faculdade de Ciências, Universidade do Porto, Rua do Campo Alegre, 4169-007 Porto, \\ Portugal \\ ${ }^{3}$ University of Zurich, Institut of computational sciences, Winterthurerstrasse 190, 8057 Zürich, Switzerland \\ 4 Physikalisches Institut, University of Bern, Gesellschaftsstrasse 6, 3012 Bern, Switzerland \\ 5 Department of Reference Systems and Geodynamics, Royal Observatory of Belgium (ROB), Avenue Circulaire 3,1180 Brussels, \\ Belgium \\ ${ }^{6}$ Institute of Geological Sciences, Freie Universität Berlin, Malteserstr. 74-100, 12249 Berlin, Germany \\ 7 Instituto de Astrofísica de Canarias, C/vía Láctea s/n, 38205 La Laguna, Tenerife, Spain \\ 8 Universidad de La Laguna, Dept. Astrofísica, 38206 La Laguna, Tenerife, Spain
}

Received 13 June 2017 / Accepted 27 October 2017

\section{ABSTRACT}

\begin{abstract}
Context. The chemical composition of stars that have orbiting planets provides important clues about the frequency, architecture, and composition of exoplanet systems.

Aims. We explore the possibility that stars from different galactic populations that have different intrinsic abundance ratios may produce planets with a different overall composition.

Methods. We compiled abundances for $\mathrm{Fe}, \mathrm{O}, \mathrm{C}, \mathrm{Mg}$, and $\mathrm{Si}$ in a large sample of solar neighbourhood stars that belong to different galactic populations. We then used a simple stoichiometric model to predict the expected iron-to-silicate mass fraction and water mass fraction of the planet building blocks, as well as the summed mass percentage of all heavy elements in the disc.

Results. Assuming that overall the chemical composition of the planet building blocks will be reflected in the composition of the formed planets, we show that according to our model, discs around stars from different galactic populations, as well as around stars from different regions in the Galaxy, are expected to form rocky planets with significantly different iron-to-silicate mass fractions. The available water mass fraction also changes significantly from one galactic population to another.

Conclusions. The results may be used to set constraints for models of planet formation and chemical composition. Furthermore, the results may have impact on our understanding of the frequency of planets in the Galaxy, as well as on the existence of conditions for habitability.
\end{abstract}

Key words. stars: abundances - planetary systems - planets and satellites: composition - techniques: spectroscopic

\section{Introduction}

The study of stars hosting planets is providing a huge amount of information about the processes of planetary formation and evolution (see e.g. Mayor et al. 2014). The dependence on the frequency of planets with the stellar metallicity and mass (e.g. Santos et al. 2004; Fischer \& Valenti 2005; Johnson et al. 2007; Sousa et al. 2011b; Buchhave et al. 2012), for example, has been suggested as strong evidence in favor of the hypothesis of the core-accretion model as the dominant giant planet formation process (e.g. Mordasini et al. 2012a). The architecture of planetary systems and its dependence on the metal content of the stars further provides indications about the processes involved in the planet migration (e.g. Dawson \& Murray-Clay 2013; Adibekyan et al. 2013b; Beaugé \& Nesvorný 2013).

The stars we observe in the solar neighbourhood can be divided into three galactic populations: the thin-disc, the thickdisc, and the halo population. Most stars are members of the younger thin-disc component, ranging in $[\mathrm{Fe} / \mathrm{H}]$ from $\sim-0.8$ up to $\sim+0.5$ dex. Thick-disc stars typically have lower metallicities than their thin-disc counterparts. More specifically, they present higher values of $\alpha$-element ${ }^{1}$ abundances (for a recent paper, see Adibekyan et al. 2013a). Finally, halo stars are usually objects of lower metallicity, which also often present $\alpha$ element enhancement. They are commonly identified using dynamical approaches (Bensby et al. 2003). The kinematical and chemical properties (in particular the abundance ratios) of these three populations reflect their origin, age, and the galactic formation process (e.g. Haywood et al. 2013); see Appendix A for more details.

Recent studies suggest that the abundances of specific chemical species in the stellar photosphere may give clues about the internal structure and composition of the planets. This is true both for giant planets (Guillot et al. 2006; Fortney et al. 2007) and for their rocky counterparts (e.g. Bond et al. 2010; Delgado Mena et al. 2010; Dorn et al. 2015; Dorn et al. 2017; Thiabaud et al. 2015; Santos et al. 2015). Stars from different

1 Elements for which the most abundant isotopes are integer multiples of 4 , the mass of a helium nucleus ( $\alpha$ particle). 
galactic populations may thus present different planet frequencies, and the planets orbiting them may present different composition trends (e.g. Haywood 2008; Adibekyan et al. 2012a, 2015, 2016a; Frank et al. 2014).

In this context, the present paper investigates whether stars that come from different galactic populations and have different chemical composition are expected to form planet building blocks (or planets) with different compositions. In Sect. 2 we present the data selected for this study, including detailed chemical abundances for several elements. In Sect. 3 we then describe our model, applying it to the stars in different galactic populations in Sect. 4. Finally, in Sect. 5 we discuss our results in face of the expected planet populations in the galaxy, including the prospects for life-bearing worlds.

\section{Data}

To explore the effect of different initial stellar abundances on the planet composition, we need to define a sample of stars for which precise abundances have been determined. As we show below, our model needs abundance values for $\mathrm{Fe}, \mathrm{Si}, \mathrm{Mg}, \mathrm{C}$, and $\mathrm{O}$ as input. To build this sample, we started from the work of Adibekyan et al. (2012b). This study, based on spectra with high signal-to-noise ratios and high resolution obtained with the HARPS spectrograph, provides abundances of $\mathrm{Fe}, \mathrm{Si}$, and $\mathrm{Mg}$ for 1111 stars in a volume-limited sample. Oxygen abundances were then added from the study of Bertran de Lis et al. (2015). The values derived using the $6158 \AA$ oxygen line were preferred, as these have been shown by the authors to be more precise. Finally, for $\mathrm{C}$ we used the carbon values recently derived by Suárez-Andrés et al. (2017). All these abundances were derived based on same set of uniform stellar atmospheric parameters (namely $T_{\text {eff }}$ and $\log g$, from Sousa et al. 2008, 2011b,a).

All abundances listed in these papers (as well as the stellar parameter analysis) were computed relative to the Sun. They were transformed into absolute abundances assuming the solar composition as given in Asplund et al. (2009) for $\mathrm{Fe}(\log \epsilon=$ 7.50), $\mathrm{Mg}(\log \epsilon=7.60)$, and $\mathrm{Si}(\log \epsilon=7.51)$, and as given by Bertran de Lis et al. (2015) and Suárez-Andrés et al. (2017) for $\mathrm{O}(\log \epsilon=8.71)$ and $\mathrm{C}(\log \epsilon=8.50)^{2}$. For helium, the adopted value was taken from Lodders $(2003, \log \epsilon=10.93)$.

In total, we have 535 stars for which precise values of abundances for $\mathrm{Fe}, \mathrm{Si}, \mathrm{Mg}, \mathrm{C}$, and $\mathrm{O}$ have been obtained. These stars represent the solar neighbourhood sample well. In order to avoid strong systematic effects or errors in the abundances caused by uncertainties in the line-lists and model atmospheres as we move away from the solar values (see e.g. Adibekyan et al. 2012b; Bertran de Lis et al. 2015), we decided to further cut our sample to include only stars with a temperature of $\pm 300 \mathrm{~K}$ around solar (we assume $T_{\text {eff }}(\odot)=5777 \mathrm{~K}$ ). This left us with 371 stars.

To classify the stars into different galactic populations, we used the chemical boundary discussed in Adibekyan et al. (2012b). According to this, 303 of the 371 stars belong to the thin disc, while 68 have enhanced $\alpha$-element abundances. This latter pattern is typical of thick-disc stars of our Galaxy. However, for $\alpha$-rich metal-rich stars $([\mathrm{Fe} / \mathrm{H}]>-0.2 \mathrm{dex})$, there is no consensus about which galactic population they belong to (Adibekyan et al. 2011; Bensby et al. 2014). We therefore divided the 68 stars into two different groups: the thick-disc

\footnotetext{
2 The value $[\mathrm{X} / \mathrm{H}]$, where $\mathrm{X}$ is a specific element, is defined as $[\mathrm{X} / \mathrm{H}]=$ $\log \frac{N_{\mathrm{X}}}{N_{\mathrm{H}}}-\log \left(\frac{N_{\mathrm{X}}}{N_{\mathrm{H}}}\right)$, where $N$ is the number of atoms. The values of $\log \epsilon$ are defined as $\log \epsilon=\log \frac{N_{\mathrm{X}}}{N_{\mathrm{H}}}+12$.
}

population (48 stars with $[\mathrm{Fe} / \mathrm{H}]<-0.2$ dex) and its $\alpha$-rich metal-rich counterpart (20 stars with $[\mathrm{Fe} / \mathrm{H}] \geq-0.2$ dex), hereafter called homr. Finally, 3 stars in our sample were identified as belonging to the galactic halo following the kinematic criteria of Bensby et al. (2003). They were treated separately since halo stars in the solar neighbourhood are also known to be mainly rich in $\alpha$ elements (but see Nissen \& Schuster 2010).

\section{Model}

The model we used here is the same as was used in Santos et al. (2015). In brief, it makes use of the abundances of the rockforming elements $\mathrm{Fe}, \mathrm{Si}, \mathrm{Mg}, \mathrm{C}$, and $\mathrm{O}$, together with $\mathrm{H}$ and $\mathrm{He}$, and assumes that these are the most relevant to control the species expected from equilibrium condensation models (Lodders 2003; Seager et al. 2007), such as $\mathrm{H}_{2}, \mathrm{He}, \mathrm{H}_{2} \mathrm{O}$, $\mathrm{CH}_{4}, \mathrm{Fe}, \mathrm{MgSiO}_{3}, \mathrm{Mg}_{2} \mathrm{SiO}_{4}$, and $\mathrm{SiO}_{2}{ }^{3}$. In other words, in our model we only include the mineral phases of the main rock-forming elements that dominate the crust, the upper and lower mantle, and the core of an Earth-like planet interior (see e.g. McDounough \& Sun 1995; Sotin et al. 2007). A simplified model for the expected mass fractions of different compounds using these species is thus a reasonable approach. In this case, the molecular abundances and therefore the mass fraction can be found from the atomic abundances with simple stoichiometry, as discussed in Santos et al. (2015); see also Bond et al. (2010), Thiabaud et al. (2015), Unterborn \& Panero (2017).

We note that no star in our sample has values of $\mathrm{Mg} / \mathrm{Si}>2$, in which case, $\mathrm{Si}$ would be incorporated in olivine and the remaining $\mathrm{Mg}$ would enter in other minerals, mostly oxides. Our simple model does not take these cases into consideration. Moreover, ten stars were found to have $\mathrm{C} / \mathrm{O}$ ratios above 0.8 : above this value, the mineralogy is expected to be significantly different (Bond et al. 2010), with carbides forming instead of the silicates; planet building blocks would then be strongly enriched in carbon. Since only one star was found with $\mathrm{C} / \mathrm{O}$ above 1 $(\mathrm{C} / \mathrm{O}=1.14)$, and given the typical (high) errors in the derivation of abundances for these species (see Bertran de Lis et al. 2015; Suárez-Andrés et al. 2017), we decided to keep these stars in the sample. In any case, observations suggest that only a small fraction of the stars in the solar neighbourhood have $\mathrm{C} / \mathrm{O}>0.8$ (Fortney 2012; Brewer \& Fischer 2016).

The simple relations presented in Santos et al. (2015; see also Appendix B) allow us to compute the expected mass fractions, in particular, the iron-to-silicate mass fraction $\left(f_{\text {iron }}\right)^{4}$, the water mass fraction $\left(w_{f}\right)$, and the summed mass percent of all heavy elements $(Z)$ expected for the planetary building blocks/grains:

$$
\begin{aligned}
f_{\text {iron }}= & m_{\mathrm{Fe}} /\left(m_{\mathrm{Fe}}+m_{\mathrm{MgSiO} 3}+m_{\mathrm{Mg} 2 \mathrm{SiO} 4}+m_{\mathrm{SiO} 2}\right) \\
w_{f}= & m_{\mathrm{H} 2 \mathrm{O}} /\left(m_{\mathrm{H} 2 \mathrm{O}}+m_{\mathrm{Fe}}+m_{\mathrm{MgSiO} 3}+m_{\mathrm{Mg} 2 \mathrm{SiO} 4}+m_{\mathrm{SiO} 2}\right) \\
Z=( & m_{\mathrm{CH} 4}+m_{\mathrm{H} 2 \mathrm{O}}+m_{\mathrm{Fe}}+m_{\mathrm{MgSiO} 3} \\
& \left.+m_{\mathrm{Mg} 2 \mathrm{SiO} 4}+m_{\mathrm{SiO} 2}\right) / M_{\mathrm{tot}},
\end{aligned}
$$

where $m_{\mathrm{X}}=N_{x} * \mu_{\mathrm{X}}, M_{\mathrm{tot}}=N_{\mathrm{H}} * \mu_{\mathrm{H}}+N_{\mathrm{He}} * \mu_{\mathrm{He}}+N_{\mathrm{C}} * \mu_{\mathrm{C}}+$ $N_{\mathrm{O}} * \mu_{\mathrm{O}}+N_{\mathrm{Mg}} * \mu_{\mathrm{Mg}}+N_{\mathrm{Si}} * \mu_{\mathrm{Si}}+N_{\mathrm{Fe}} * \mu_{\mathrm{Fe}}$, and $N_{\mathrm{X}}$ represents the number of atoms of each species $\mathrm{X}$, and $\mu_{\mathrm{X}}$ their mean molecular weights. All $N_{\mathrm{X}}$ values are computed relative to hydrogen.

These variables are expected to have an important influence on the planet structure and radius (Dorn et al. 2017). They are

\footnotetext{
3 There are more silicate forms, but these are the most relevant.

4 Not taking into account the iron silicates.
} 
N. C. Santos et al.: Constraining planet structure and composition from stellar chemistry: trends in different stellar populations

Table 1. Average values and standard deviations for $f_{\text {iron }}$ and the water mass fraction in the different galactic populations.

\begin{tabular}{lcc}
\hline \hline Population & Average & Std. \\
\hline$f_{\text {iron }}$ & & \\
Thin disc & 31.974 & 1.750 \\
Thin disc $([\mathrm{Fe} / \mathrm{H}]<-0.2)$ & 29.430 & 1.550 \\
Thin disc $([\mathrm{Fe} / \mathrm{H}] \geq-0.2)$ & 32.514 & 1.240 \\
Thick disc $([\mathrm{Fe} / \mathrm{H}]<-0.2)$ & 22.464 & 1.742 \\
h $\alpha$ mr & 28.727 & 1.338 \\
Halo & 23.110 & 2.884 \\
\hline$w_{f}$ & & \\
Thin disc & 59.713 & 7.106 \\
Thin disc $([\mathrm{Fe} / \mathrm{H}]<-0.2)$ & 68.014 & 7.116 \\
Thin disc $([\mathrm{Fe} / \mathrm{H}] \geq-0.2)$ & 57.953 & 5.724 \\
Thick disc $([\mathrm{Fe} / \mathrm{H}]<-0.2)$ & 76.172 & 5.448 \\
h $\alpha$ mr & 62.591 & 7.194 \\
Halo & 83.990 & 4.115 \\
\hline$Z$ & & \\
Thin disc & 1.508 & 0.597 \\
Thin disc $([\mathrm{Fe} / \mathrm{H}]<-0.2)$ & 0.818 & 0.181 \\
Thin disc $([\mathrm{Fe} / \mathrm{H}] \geq-0.2)$ & 1.655 & 0.550 \\
Thick disc $([\mathrm{Fe} / \mathrm{H}]<-0.2)$ & 0.870 & 0.233 \\
h $\alpha$ mr & 1.773 & 0.444 \\
Halo & 0.808 & 0.082 \\
\hline
\end{tabular}

Notes. ${ }^{(\dagger)}$ We only have three halo stars, which limits the conclusions for this specific galactic population.

also expected to provide relevant information about some parameters relevant for the planet formation process, such as the mass fraction of all heavy elements and the ice mass fraction in solid planets, or giant planet cores that form beyond the water ice-line.

We should note that in the calculations above we assume that the composition of $\mathrm{H}, \mathrm{C}, \mathrm{N}, \mathrm{O}, \mathrm{Mg}, \mathrm{Si}$, and $\mathrm{Fe}$ dominates the composition of the solid material. This is likely a good approximation, as in the Sun these elements represent the main contributors to the overall composition (see abundances in e.g. Asplund et al. 2009).

The model predicts that planet building blocks for solar system have $f_{\text {iron }}=33 \%, w_{f}=60 \%$, and $Z=1.26 \%$. The value of $f_{\text {iron }}$ is compatible to the value observed in the terrestrial planets (see the discussion below), while $w_{f}$ and $Z$ are similar to the values derived in Lodders (2003, 67.11\% and $1.31 \%$, respectively).

In Appendix $\mathrm{C}$ we present some simple relations to compute the values of $f_{\text {iron }}$ and $Z$ based on the stellar abundances.

\section{Results}

In Fig. 1 we present the distributions of iron and water mass fractions (top and middle panels) for the stars in our sample, as derived with the model described above. The bottom panels present the values of $Z$. The left panels present the comparison of the four galactic populations mentioned above, and the right panels present the comparison between thin-disc stars of different metallicity. This separation is motivated by the fact that low-metallicity thin-disc stars present an increasing $\alpha$-element enhancement as $[\mathrm{Fe} / \mathrm{H}]$ decreases (e.g. Adibekyan et al. 2012a). In Table 1 we present the average and standard deviation values for the different populations.
The results presented in Fig. 1 and in the table show that (on average) we can expect planet building blocks in different galactic populations to have significantly different values of iron and water mass fraction. Average values of $f_{\text {iron }}$ over the different populations vary between 22.5 and 23.1 for the metal-poor thick-disc and halo stars, respectively, and rise up to 32.5 for the metal-rich thin-disc population. Water mass fractions also vary from average values of $\sim 58 \%$ for the thin-disc metal-rich stars to $76 \%$ and $83 \%$ for the metal-poor thick-disc and halo.

Several conclusions can be drawn from comparing different groups of thin-disc stars. First, and as expected, the value of $f_{\text {iron }}$ and $w_{f}$ for stars of the metal-rich thin-disc population (where the Sun is included) suggest that these should be able to host planets similar in composition to the solar system planets. Metal-poor thin-disc stars, on the other hand, are expected to have lower iron and higher water mass fractions than the fractions expected for the Sun. The relatively small increase in the water fraction is related to the relatively higher $[\mathrm{O} / \mathrm{Fe}]$ ratios observed in metalpoor thin-disc stars (Bertran de Lis et al. 2015) when compared to their metal-rich counterparts. The lower $f_{\text {iron }}$ reflects the decrease in metallicity and increase in the $\alpha$-element abundances.

According to our results, the metal-poor thick-disc population is expected to produce planets with much lower iron mass fractions (that may be translated into smaller cores). The water mass fraction in these stars, however, is higher than the fraction found in thin-disc objects. This is again expected as these stars present on average higher oxygen abundances than thindisc stars of the same $[\mathrm{Fe} / \mathrm{H}]$. Interestingly, our results also suggest that homr stars might produce planets with values of $f_{\text {iron }}$ and $w_{f}$ intermediate between the thin-disc and the metal-poor thick-disc stars. This reflects their high $\alpha$-element abundances $(\mathrm{O}, \mathrm{C}, \mathrm{Si}$, and $\mathrm{Mg}$ ) when compared to thin-disc stars of the same metallicity, which leads to a relative increase in water mass fraction but a decrease of $f_{\text {iron }}$.

We only have three halo stars in our sample, which limits our conclusions regarding this population. However, and as expected, our model indicates that they might produce planets with a low iron mass fraction while being water rich. To our knowledge, there is no planet detected so far that orbits a halo star.

Finally, the average values of $Z$ for the different populations range from $\sim 0.8 \%$ to $\sim 1.7 \%$ (a factor of $\sim 2$ ), with the individual values ranging from $\sim 0.5 \%$ up to $\sim 3 \%$. As expected, metalpoor thin-disc and halo stars have the lowest average values of $Z$, while the remaining populations have higher values, even if a large spread is observed in each population. $Z$ is expected to be related to the planet formation efficiency. Increasing $Z$ may increase the probability of forming terrestrial-like planets or the icy/rocky cores of giant planets. This fact is behind the usual interpretation for the clear metallicity-giant planet correlation that is observed (e.g. Santos et al. 2004; Fischer \& Valenti 2005). Interestingly, even if such a clear correlation has not been found for stars hosting the lower mass planets (e.g. Sousa et al. 2011b; Buchhave et al. 2012), hints exists that the overall metallicity still plays a role in this planet mass regime (e.g. Adibekyan et al. 2012a; Wang \& Fischer 2015; Zhu et al. 2016).

\section{Discussion: planet composition, frequency, and habitability}

The results presented above suggest that solar neighbourhood stars from different galactic populations whose abundance ratios are different may be able to produce planets, or at least 

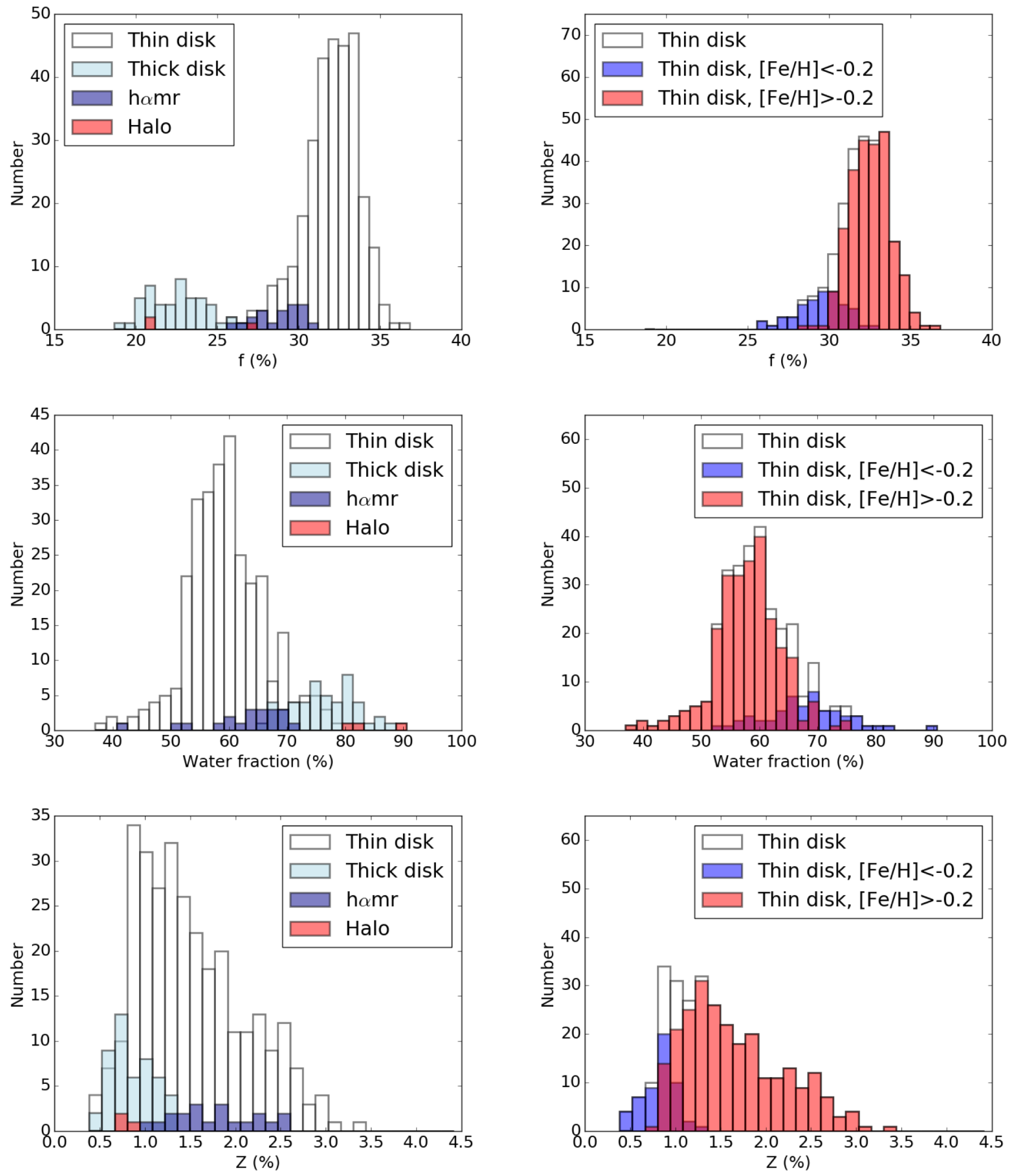

Fig. 1. $f_{\text {iron }}$ (upper panels), $w_{f}$ (middle panels), and $Z$ (lower panels) mass fractions distributions for the different populations in the Galaxy.

the planet building blocks, that have different intrinsic compositions. Thick-disc stars in our Galaxy, for example, are known to be older than their thin-disc counterparts (e.g. Haywood et al. 2013). Stars in different galactic regions (inner vs. outer disk and stars with different heights above or below the galactic plane) also present different abundance ratios (e.g. Hayden et al. 2015; Haywood et al. 2016). As has been discussed in some recent works (e.g. Frank et al. 2014; Adibekyan et al. 2016a,b), this may imply that planets formed in different galactic ages (past or future) or in different galactic regions may have a 
significantly different composition trend. The planet formation efficiency around different populations of stars may also be different (Adibekyan et al. 2012a).

Understanding whether the initial conditions for planet formation depend on the galactic population can also constrain the modelling of planet formation and planet structure. For instance, the overall amount of solid material $(Z)$ and the expected composition of the building blocks $\left(f_{\text {iron }}\right.$ and $\left.w_{f}\right)$ can be used as a prior to understand the frequency and populations of planets existing in the solar neighbourhood from planet population synthesis models (e.g. Mordasini et al. 2012b). Furthermore, priors on the planet composition can be set when modelling a planet based on its mass-radius relation (e.g. Dorn et al. 2017), as long as the abundances for some elements are known in the host star, or if the stellar population is deduced from kinematic studies (e.g. with Gaia), for example.

We note that our basic assumption is that the chemical composition of the (rocky) planets is intimately related to the chemical composition of the star. This is likely a good approximation from a statistical approach, as in the solar system the relative amount of refractory elements is correlated between meteorites, the solar photosphere, and the terrestrial planets Earth, Venus, and Mars (e.g. Morgan \& Anders 1980; Lodders 2003; Drake \& Righter 2002; Lodders \& Fegley 1998; Khan \& Connolly 2008; Sanloup et al. 1999). This is also found from models (see e.g. Thiabaud et al. 2015), even if exceptions may exist (Dorn et al. 2017). Finally, observations of rocky exoplanets also seem to suggest that they tend to follow the Earth composition line (e.g. Motalebi et al. 2015), in agreement with the assumptions taken here (see also Santos et al. 2015). In this context, it would now be interesting to understand, for instance, if the mentioned result is compatible with the radius of planets orbiting metal-poor thick-disc stars (e.g. EPIC 210894022b/K2-111b - Fridlund et al. 2017). Exceptions to this trend may also have been found. These can be the cases of LHS 1140 b (Dittmann et al. 2017), for which the best-fit model implies a higher core mass fraction (even if compatible with Earth-like fractions within the errors), or the recently discovered very short period K2-106 b (Guenther et al. 2017). On the positive side, recent results suggest that the star TRAPPIST-1 may be a thick-disc object (Burgasser \& Mamajek 2017). Curiously, the planets discovered in this system that have a good mass estimate have been proposed to have a low density (favouring a volatilerich composition - Gillon et al. 2017), a result compatible with our model. It would be very interesting to investigate the detailed abundances of these specific stars in face of the results obtained above. New planet detections around bright nearby stars, expected with missions such as TESS, CHEOPS, and PLATO, will certainly allow us to address this field.

We also assume here that no internal or external physical process are able to significantly alter the initial stellar composition. Planet engulfment processes have been suggested to be responsible for specific stellar abundance patterns (Israelian et al. 2001), even if such events may be rare. Furthermore, the magnitude of this effect on the stellar abundances of G-type stars (with deep convective envelopes) is expected to be small when compared with the typical uncertainties in the abundances (e.g. Santos et al. 2003). Other less well understood effects, such as element diffusion, could also affect specific stellar abundances (e.g. Önehag et al. 2014), but the magnitude of this effect is in any case expected to be small for solar-type stars (Önehag et al. 2014; Gonzalez 2014). These effects need to be kept in mind, however, even if they may only be able to introduce minor changes to the original chemical abundances.
We also stress that the results discussed here can be used to estimate the composition of the planet building blocks, but they might not be directly applicable to the final composition or internal structure of a final differentiated planet (or chemical distribution within the planet). The composition of a planet of course depends on the region of the proto-planetary disc where it formed as well as on its migration path (e.g. Mordasini et al. 2012b; Carter-Bond et al. 2012). Physical processes, including evaporation and collisional stripping, may also be able to change the overall composition of a planet (including its coreto-mantle fraction), as likely happened to Mercury (Benz et al. 1988; Marcus et al. 2010a). Moreover, the compositional evolution of a planet depends on the accretion and differentiation history for which the understanding of the compositional model and the cooling history of the primitive body are key (Rubie et al. 2015; Fischer et al. 2017).

Regarding the water content, and given the spread in water fractions in our solar system's terrestrial planets, we should also add that the origin of the water in our own planet Earth is still strongly debated, and several external processes (e.g. comet infall or strong stellar activity) that occurred during and after planet formation may likely significantly change the amount of water on the surface of a planet (Marcus et al. 2010b; Luger et al. 2015). The values of $w_{f}$ derived are therefore expected to be indicative of the amount of water available in the proto-planetary disc; although this probably correlates with the final water content of the planets, strong scatter is expected. Water accumulates in the outer part of the disc during the later condensation of volatile atoms compared to heavier elements such as silicates and iron, which make up the main building blocks of the inner part of the proto-planetary disc. Even though the outer planets Jupiter, Saturn, Uranus, and Neptune contain mostly hydrogen, helium, and methane above their rocky core, the compositions of several of their moons are dominated by water and ice. The amount of available water can also be related to the planet (or core) formation efficiency beyond the ice line, as it will control the amount of condensates in this region.

Overall, our results suggest that stars in different galactic populations may produce planets with different core mass fractions and water contents. It is thus interesting to discuss the implications of this result for astrobiology. It has been suggested that large core mass fractions may inhibit plate tectonics (Noack et al. 2014). Plate tectonics is believed to be important for the evolution of complex life since it helps to maintain the magnetic field, replenishes the surface with nutrient-rich soil, and stabilizes the long-term global carbon cycle. Furthermore, in the absence of plate tectonics, large cores can suppress outgassing of greenhouse gases on stagnant-lid planets (Noack et al. 2017). Finally, water has also been discussed to be a key ingredient for promoting plate tectonics (Korenaga 2010) and plays a crucial role for the surface habitability of rocky planets as well as for their interior evolution. Water in the mantle significantly reduces both silicate melting temperatures and mantle viscosity, and also influences the chemistry in the mantle. Different planet compositions may in this context alter the width of the habitable zones, since plate tectonics is related to the capacity to degass $\mathrm{CO}_{2}$. This may have implications for the definition of the so-called Galactic habitable zone (e.g. Lineweaver et al. 2004; Prantzos 2008; Carigi et al. 2013; Gonzalez 2014; Spitoni et al. 2017). Since the quantity of available water in a planet may be related to the relative water content existing in the planet building blocks, the values derived in this paper may also give us some indications about the prevalence of water worlds (Léger et al. 2004; Simpson 2017). 
Acknowledgements. This work was supported by Fundação para a Ciência e a Tecnologia (FCT, Portugal) through the research grant through national funds and by FEDER through COMPETE2020 by grants UID/FIS/04434/2013 \& POCI-01-0145-FEDER-007672, PTDC/FIS-AST/1526/2014 \& POCI-01-0145FEDER-016886 and PTDC/FIS-AST/7073/2014 \& POCI-01-0145- FEDER016880. P.F., S.B., N.C.S. e S.G.S. acknowledge support from FCT through Investigador FCT contracts No. IF/01037/2013CP1191/CT0001, IF/ 01312/2014/CP1215/CT0004, IF/00169/2012/CP0150/CT0002, and IF/00028/ 2014/CP1215/CT0002. V.A. and E.D.M. acknowledge support from FCT through Investigador FCT contracts No. IF/00650/ 2015/CP1273/CT0001, IF/00849/2015/CP1273/CT0003 and by the fellowship SFRH/BPD/70574/2010, SFRH/BPD/76606/2011 funded by FCT and POPH/FSE (EC). PF further acknowledges support from Fundação para a Ciência e a Tecnologia (FCT) in the form of an exploratory project of reference IF/01037/2013CP1191/CT0001. L.N. has been funded by the Interuniversity Attraction Poles Programme initiated by the Belgian Science Policy Office through the Planet Topers alliance and by the Deutsche Forschungsgemeinschaft (SFB-TRR 170). C.M. acknowledges the support from the Swiss National Science Foundation under grant BSSGI0 155816 "PlanetsInTime". Parts of this work have been carried out within the frame of the National Center for Competence in Research PlanetS supported by the SNSF. This work results within the collaboration of the COST Action TD 1308

\section{References}

Adibekyan, V. Z., Santos, N. C., Sousa, S. G., \& Israelian, G. 2011, A\&A, 535, L11

Adibekyan, V. Z., Delgado Mena, E., Sousa, S. G., et al. 2012a, A\&A, 547, A36 Adibekyan, V. Z., Sousa, S. G., Santos, N. C., et al. 2012b, A\&A, 545, A32 Adibekyan, V. Z., Figueira, P., Santos, N. C., et al. 2013a, A\&A, 554, A44 Adibekyan, V. Z., Figueira, P., Santos, N. C., et al. 2013b, A\&A, 560, A51 Adibekyan, V., Santos, N. C., Figueira, P., et al. 2015, A\&A, 581, L2 Adibekyan, V., Delgado-Mena, E., Figueira, P., et al. 2016a, A\&A, 592, A87 Adibekyan, V., Figueira, P., \& Santos, N. C. 2016b, Origins of Life and Evolution of the Biosphere, 46, 351

Asplund, M., Grevesse, N., Sauval, A. J., \& Scott, P. 2009, ARA\&A, 47, 481

Beaugé, C., \& Nesvorný, D. 2013, ApJ, 763, 12

Bensby, T., Feltzing, S., \& Lundström, I. 2003, A\&A, 410, 527

Bensby, T., Feltzing, S., Lundström, I., \& Ilyin, I. 2005, A\&A, 433, 185

Bensby, T., Feltzing, S., \& Oey, M. S. 2014, A\&A, 562, A71

Benz, W., Slattery, W. L., \& Cameron, A. G. W. 1988, Icarus, 74, 516

Bertran de Lis, S., Delgado Mena, E., Adibekyan, V. Z., Santos, N. C., \& Sousa S. G. 2015, A\&A, 576, A89

Bond, J. C., O’Brien, D. P., \& Lauretta, D. S. 2010, ApJ, 715, 1050

Bovy, J., Rix, H.-W., Liu, C., et al. 2012, ApJ, 753, 148

Brewer, J. M., \& Fischer, D. A. 2016, ApJ, 831, 20

Buchhave, L. A., Latham, D. W., Johansen, A., et al. 2012, Nature, 486, 375

Burgasser, A. J., \& Mamajek, E. E. 2017, ApJ, 845, 110

Buser, R. 2000, Science, 287, 69

Carigi, L., García-Rojas, J., \& Meneses-Goytia, S. 2013, Rev. Mex. Astron. Astrofis., 49, 253

Carter-Bond, J. C., O’Brien, D. P., \& Raymond, S. N. 2012, ApJ, 760, 44

Dawson, R. I., \& Murray-Clay, R. A. 2013, ApJ, 767, L24

Delgado Mena, E., Israelian, G., González Hernández, J. I., et al. 2010, ApJ, 725, 2349

Dittmann, J. A., Irwin, J. M., Charbonneau, D., et al. 2017, Nature, 544, 333

Dorn, C., Khan, A., Heng, K., et al. 2015, A\&A, 577, A83

Dorn, C., Hinkel, N. R., \& Venturini, J. 2017, A\&A, 597, A38

Drake, M. J., \& Righter, K. 2002, Nature, 416, 39

Fischer, D. A., \& Valenti, J. 2005, ApJ, 622, 1102

Fischer, R. A., Campbell, A. J., \& Ciesla, F. J. 2017, Earth Planet. Sci. Lett., 458, 252

Fortney, J. J. 2012, ApJ, 747, L27

Fortney, J. J., Marley, M. S., \& Barnes, J. W. 2007, ApJ, 659, 1661

Frank, E. A., Meyer, B. S., \& Mojzsis, S. J. 2014, Icarus, 243, 274

Fridlund, M., Gaidos, E., Barragán, O., et al. 2017, A\&A, 604, A16

Fuhrmann, K. 2008, MNRAS, 384, 173
Gillon, M., Triaud, A. H. M. J., Demory, B.-O., et al. 2017, Nature, 542, 456 Gilmore, G., \& Reid, N. 1983, MNRAS, 202, 1025

Gonzalez, G. 2014, MNRAS, 443, 393

Guenther, E. W., Barragan, O., Dai, F., et al. 2017, A\&A, 608, A93

Guillot, T., Santos, N. C., Pont, F., et al. 2006, A\&A, 453, L21

Hayden, M. R., Bovy, J., Holtzman, J. A., et al. 2015, ApJ, 808, 132

Haywood, M. 2008, A\&A, 482, 673

Haywood, M., Di Matteo, P., Lehnert, M. D., Katz, D., \& Gómez, A. 2013, A\&A, 560, A109

Haywood, M., Lehnert, M. D., Di Matteo, P., et al. 2016, A\&A, 589, A66

Israelian, G., Santos, N. C., Mayor, M., \& Rebolo, R. 2001, Nature, 411, 163

Ivezić, Ž., Beers, T. C., \& Jurić, M. 2012, ARA\&A, 50, 251

Johnson, J. A., Butler, R. P., Marcy, G. W., et al. 2007, ApJ, 670, 833

Jurić, M., Ivezić, Ž., Brooks, A., et al. 2008, ApJ, 673, 864

Khan, A., \& Connolly, J. A. D. 2008, J. Geophys. Res.: Planets, 113, e07003

Kordopatis, G., Wyse, R. F. G., Gilmore, G., et al. 2015, A\&A, 582, A122

Korenaga, J. 2010, ApJ, 725, L43

Léger, A., Selsis, F., Sotin, C., et al. 2004, Icarus, 169, 499

Lineweaver, C. H., Fenner, Y., \& Gibson, B. K. 2004, Science, 303, 59

Lodders, K. 2003, ApJ, 591, 1220

Lodders, K., \& Fegley, B. 1998, The planetary scientist's companion/Katharina Lodders, Bruce Fegley (New York: Oxford University Press)

Luger, R., Barnes, R., Lopez, E., et al. 2015, Astrobiology, 15, 57

Marcus, R. A., Sasselov, D., Hernquist, L., \& Stewart, S. T. 2010a, ApJ, 712, L73

Marcus, R. A., Sasselov, D., Stewart, S. T., \& Hernquist, L. 2010b, ApJ, 719, L45

Mayor, M., Lovis, C., \& Santos, N. C. 2014, Nature, 513, 328

McDounough, W. F., \& Sun, S.-s. 1995, Chem. Geol., 120, 223

Mordasini, C., Alibert, Y., Benz, W., Klahr, H., \& Henning, T. 2012a, A\&A, 541, A97

Mordasini, C., Alibert, Y., Georgy, C., et al. 2012b, A\&A, 547, A112

Morgan, J. W., \& Anders, E. 1980, Proc. National Academy of Science, 77, 6973

Motalebi, F., Udry, S., Gillon, M., et al. 2015, A\&A, 584, A72

Navarro, J. F., Abadi, M. G., Venn, K. A., Freeman, K. C., \& Anguiano, B. 2011, MNRAS, 412, 1203

Nissen, P. E., \& Schuster, W. J. 2010, A\&A, 511, L10

Noack, L., Godolt, M., von Paris, P., et al. 2014, Planet. Space Sci., 98, 14

Noack, L., Rivoldini, A., \& Hoolst, T. V. 2017, Physics of the Earth and Planetary Interiors, 269, 40

Önehag, A., Gustafsson, B., \& Korn, A. 2014, A\&A, 562, A102

Prantzos, N. 2008, Space Sci. Rev., 135, 313

Recio-Blanco, A., de Laverny, P., Kordopatis, G., et al. 2014, A\&A, 567, A5

Rubie, D., Jacobson, S., Morbidelli, A., et al. 2015, Icarus, 248, 89

Sanloup, C., Jambon, A., \& Gillet, P. 1999, Physics of the Earth and Planetary Interiors, 112,43

Santos, N. C., Israelian, G., Mayor, M., Rebolo, R., \& Udry, S. 2003, A\&A, 398, 363

Santos, N. C., Israelian, G., \& Mayor, M. 2004, A\&A, 415, 1153

Santos, N. C., Adibekyan, V., Mordasini, C., et al. 2015, A\&A, 580, L13

Seager, S., Kuchner, M., Hier-Majumder, C. A., \& Militzer, B. 2007, ApJ, 669, 1279

Simpson, F. 2017, MNRAS, 468, 2803

Snaith, O., Haywood, M., Di Matteo, P., et al. 2015, A\&A, 578, A87

Sotin, C., Grasset, O., \& Mocquet, A. 2007, Icarus, 191, 337

Sousa, S. G., Santos, N. C., Mayor, M., et al. 2008, A\&A, 487, 373

Sousa, S. G., Santos, N. C., Israelian, G., et al. 2011a, A\&A, 526, A99

Sousa, S. G., Santos, N. C., Israelian, G., Mayor, M., \& Udry, S. 2011b, A\&A, 533, A141

Spitoni, E., Gioannini, L., \& Matteucci, F. 2017, A\&A, 605, A38

Suárez-Andrés, L., Israelian, G., González Hernández, J. I., et al. 2017, A\&A, 599, A96

Thiabaud, A., Marboeuf, U., Alibert, Y., Leya, I., \& Mezger, K. 2015, A\&A, 580, A30

Unterborn, C. T., \& Panero, W. R. 2017, ApJ, 845, 61

Wang, J., \& Fischer, D. A. 2015, AJ, 149, 14

Zhu, W., Wang, J., \& Huang, C. 2016, ApJ, 832, 196 


\section{Appendix A: Notes on Galactic populations}

The division between the disc and halo was identified long ago, but the thick disc was discovered far more recently by Gilmore \& Reid (1983) by analysing the stellar density distribution as a function of distance from the Galactic plane. The thin- and thick-disc populations have different kinematics and chemical properties. Generally, the thick disc is thought to be composed of relatively old stars (e.g. Bensby et al. 2005; Fuhrmann 2008; Adibekyan et al. 2011) that usually are metal poor and $\alpha$-element enhanced (e.g. Adibekyan et al. 2013a; Recio-Blanco et al. 2014), and for which the stellar number density has a large scale-height and short scale-length ${ }^{5}$ (e.g. Bovy et al. 2012). Most stars in the solar neighbourhood ${ }^{6}$ are members of the younger thin-disc component, and they range in $[\mathrm{Fe} / \mathrm{H}]$ from $\sim-0.8$ up to $\sim+0.5$ dex (Kordopatis et al. 2015; Adibekyan et al. 2013a). Thick-disc and halo stars typically have lower metallicities than their thin-disc counterparts.

There is no obvious predetermined way to distinguish between different stellar populations in the solar neighbourhood. However, since chemistry is a relatively more stable property of a star than its spatial positions and kinematics, it is becoming increasingly clear that a dissection of the Galactic discs based only on stellar abundances is superior to kinematic separation (see Navarro et al. 2011; Adibekyan et al. 2011). Stellar ages can also be effectively used to separate the thin- and thick-disc stars (e.g. Haywood et al. 2013), although they are very difficult to obtain in high precision. Halo stars are commonly identified using dynamical approaches (e.g. Bensby et al. 2003) since these stars share similar chemical properties with their thick-disc counterparts. The kinematical and chemical properties (in particular the abundance ratios) of these three populations reflect their origin, age, and the galactic formation process (e.g. Ivezić et al. 2012).

We refer to Fig. 1 of Buser (2000) for a good scheme of the different Galactic populations.

\section{Appendix B: Our model equations}

Our model is based on the following stoichiometric relations. When $N_{\mathrm{Mg}}>N_{\mathrm{Si}}$ (see e.g. Bond et al. 2010; Thiabaud et al. 2015; Unterborn \& Panero 2017),

$N_{\mathrm{O}}=N_{\mathrm{H}_{2} \mathrm{O}}+3 N_{\mathrm{MgSiO}_{3}}+4 N_{\mathrm{Mg}_{2} \mathrm{SiO}_{4}}$

$N_{\mathrm{Mg}}=N_{\mathrm{MgSiO}_{3}}+2 N_{\mathrm{Mg}_{2} \mathrm{SiO}_{4}}$

$N_{\mathrm{Si}}=N_{\mathrm{MgSiO}_{3}}+N_{\mathrm{Mg}_{2} \mathrm{SiO}_{4}}$

$N_{\mathrm{C}}=N_{\mathrm{CH}_{4}}$,

otherwise, when $N_{\mathrm{Mg}} \leq N_{\mathrm{Si}}$,

$N_{\mathrm{O}}=N_{\mathrm{H}_{2} \mathrm{O}}+3 N_{\mathrm{MgSiO}_{3}}+2 N_{\mathrm{SiO}_{2}}$

$N_{\mathrm{Mg}}=N_{\mathrm{MgSiO}_{3}}$

$N_{\mathrm{Si}}=N_{\mathrm{MgSiO}_{3}}+N_{\mathrm{SiO}_{2}}$

$N_{\mathrm{C}}=N_{\mathrm{CH}_{4}}$.

Inverting these equations and adding the observed stellar abundances allows us to derive the ratios analysed in this work.

\footnotetext{
5 We note, however, that previous studies have obtained longer scalelengths for the stellar density profile of the thick disc (e.g. Jurić et al. 2008).

6 This value is around $\sim 50 \%$ if we consider the whole Galaxy (e.g. Snaith et al. 2015).
}

\section{Appendix C: Predicting the iron mass fraction}

In Fig. C. 1 (upper panel) we present the derived $f_{\text {iron }}$ for all the stars in our sample as a function of $[\mathrm{Si} / \mathrm{Fe}]$. We divided the stars into different populations, following the definition discussed in Sect. 2. The plot illustrates the dependence of our model on the different chemical abundances. In particular, it shows that $f_{\text {iron }}$ is strongly dependent on the $[\mathrm{Si} / \mathrm{Fe}]$ ratio $^{7}$. A cubic fit to the data provides the relation

$$
\begin{aligned}
f= & 253.522[\mathrm{Si} / \mathrm{Fe}]^{3}-61.149[\mathrm{Si} / \mathrm{Fe}]^{2} \\
& -53.342[\mathrm{Si} / \mathrm{Fe}]+33.240
\end{aligned}
$$

This equation can be used to predict the iron mass contents for the planetary building blocks when the values of $[\mathrm{Si} / \mathrm{Fe}]$ are known.

Similarly, in Fig. C.1 (middle panel), we plot the relation between the values of $Z$ and an index defined as

Index $_{1}=\log 10^{\epsilon(\mathrm{Mg})}+10^{\epsilon(\mathrm{Si})}+10^{\epsilon(\mathrm{Fe})}$.

Here $\epsilon(\mathrm{X})$ denotes the abundance of a given element. The choice of $\mathrm{Mg}, \mathrm{Si}$, and $\mathrm{Fe}$ to build this index comes from the fact that these are the easiest to measure in a solar-type star of the elements we studied here.

$$
\begin{aligned}
Z= & 1.596 \text { Index }_{1}^{3}-34.971 \text { Index }_{1}^{2} \\
& +255.799 \text { Index }_{1}-623.802 .
\end{aligned}
$$

This relation can be used to estimate the value of $Z$ in any solar neighbourhood star when the abundances of these three elements are known.

Finally, when the abundances for $\mathrm{C}$ and $\mathrm{O}$ are also available, we can define the index above as

Index $_{2}=\log 10^{\epsilon(\mathrm{Mg})}+10^{\epsilon(\mathrm{Si})}+10^{\epsilon(\mathrm{Fe})}+10^{\epsilon(\mathrm{C})}+10^{\epsilon(\mathrm{O})}$.

In this case, a cubic fit to the data (see Fig. C.1, lower panel) provides a much tighter relation:

$$
\begin{aligned}
Z= & 2.280 \text { Index }_{2}^{3}-57.831 \text { Index }_{2}^{2} \\
& +490.140 \text { Index }_{2}-1387.773 .
\end{aligned}
$$

\footnotetext{
7 The same correlation is also obtained using the $[\mathrm{Mg} / \mathrm{Fe}]$ ratio, as these two are tightly related. However, since there are more Si lines in the spectrum of a solar-type star, precise $\mathrm{Si}$ abundances are easier to
} obtain, and so we favour using Si here. 

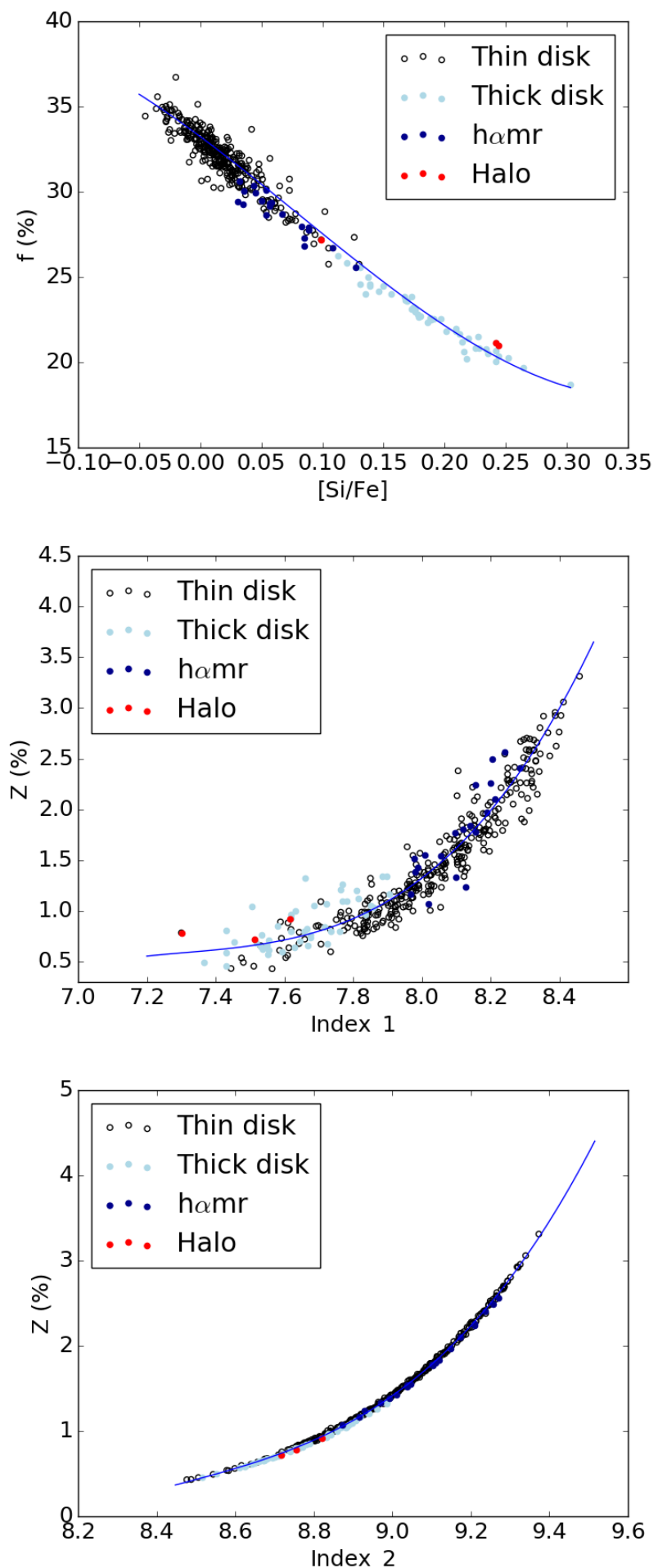

Fig. C.1. Upper panel: values of $f_{\text {iron }}$ as a function of $[\mathrm{Si} / \mathrm{Fe}]$. Middle and lower panels: $Z$ as a function of two different index values as defined in the text. Different galactic populations are denoted with different symbols. The lines denote linear cubic fits to the data. 